\title{
Saint-Georges-de-Lévejac
}

Grotte II du Valat del Malclapio

\section{Gilbert Fages}

\section{(2) OpenEdition}

\section{Journals}

Édition électronique

URL : http://journals.openedition.org/adlfi/11858

ISSN : 2114-0502

Éditeur

Ministère de la culture

Référence électronique

Gilbert Fages, «Saint-Georges-de-Lévejac », ADLFI. Archéologie de la France - Informations [En ligne], Languedoc-Roussillon, mis en ligne le 01 mars 2004, consulté le 03 mai 2019. URL : http:// journals.openedition.org/adlfi/11858

Ce document a été généré automatiquement le 3 mai 2019.

(C) Ministère de la Culture et de la Communication, CNRS 


\title{
Saint-Georges-de-Lévejac
}

\author{
Grotte II du Valat del Malclapio
}

\section{Gilbert Fages}

Date de l'opération : 1995 (SU)

Inventeur(s) : Fages Gilbert (SRA)

1 La grotte II du Valat del Malclapio est nichée dans la falaise supérieure formant corniche du versant méridional du causse de Sauveterre baigné par le Tarn. En fait, le site couronne un secteur particulièrement pittoresque des gorges au débouché aval des «Détroits» où des épandages alluviaux ont naguère fixé plusieurs exploitations agricoles : la Croze, aujourd'hui hameau de villégiature.

2 L'opération fait suite à une prospection intervenue après un incendie estival qui a ravagé le couvert sylvicole peuplé de pins sylvestres et de chênes pubescents.

3 L'accès au porche aérien se fait par une courte vire qui profite d'un joint de stratification situé à une douzaine de mètres au-dessus du pied de la falaise calcaire. Le fond du porche, au sol ascendant, conduit à un diverticule sous-cutané de $4 \mathrm{~m}$ de développement terminé par une lucarne axiale. Les matériaux détritiques externes, piégés par cette lucarne - ou fissure -, recouvrent ainsi, dans la partie terminale, l'horizon archéologique (Fig. $n^{\circ} 1$ : Topographie de la grotte du Valat del Malciapo; plan et coupes. En grisé, la zone sépulcrale.). À l'évidence, ce dernier est fort résiduel. La configuration générale (ouverture suspendue, déclivité vers l'extérieur) ne pouvait que favoriser la vidange par gravité naturelle. Dans toute la partie antérieure (porche et vestibule) le sol rougeâtre est constitué par un comblement karstique retenu par les aspérités de la roche. Contre toute attente des restes humains de petite dimension pointent de ce sédiment argileux exempt, apparemment, de traces organiques et d'indices d'anthropisation décelables par la simple observation de la teinte et de la texture.

4 Si l'objectif premier de l'intervention était de sauver les derniers éléments appelés à disparaître à brève échéance, elle se devait aussi par un dégagement et un enregistrement 
adaptés d'éclairer le fonctionnement de la sépulture et le devenir des restes au cours du temps et des aléas du milieu naturel.

5 L'essentiel de la documentation rassemblée provient de la sépulture collective du Néolithique Final/Chalcolithique (stade évolué du Groupe des Treilles tel que l'a défini G. Costantini) établie dans un espace restreint précédé d'un accès escarpé. Ce lieu a été choisi peut-être pour la sécurité qu'il procure au monde des morts par ses difficultés d'accès ou la barrière physique instaurée entre l'état des vivants et ceux qui l'ont quitté (Fig. $n^{\circ} 2$ : Le dépôt sépulcral occupe la partie reculée de la cavité, des vestiges ont migré dans le couloir d'accès en pente vers l'extérieur.). Le NMI provisoire fondé sur le décompte des os du tarse est de vingt-deux. Chiffre conciliable avec le total brut des dents : cinq cent cinquante-neuf. À côté des adultes, la présence de sujets immatures et d'enfants, dont un très jeune, est à noter. La singularité de cette sépulture est le déficit flagrant des os longs et des crânes (même fragmentés ou réduits à l'état d'esquilles) par rapport au nombre d'individus dénombrés et à la quantité des os courts et/ou petits (Fig. $n^{\circ} 3$ : Localisation des mandibules et des principaux fragments crâniens.). La fuite gravitaire ne paraît pas pouvoir tout expliquer. L'hypothèse d'un traitement particulier classement ou tri - par l'homme préhistorique peut être proposée. Plusieurs lésions pathologiques sur vertèbres, côtes, phalanges, etc. sont à signaler. Plus inhabituel pour l'époque est la relative abondance des caries et des mandibules édentées (alvéoles cicatrisés ou en voie d'occlusion). Il serait intéressant de retrouver la cause de ce mauvais état sanitaire.

Le mobilier archéologique (Fig. $\mathrm{n}^{\circ} 4$ : Plan de localisation des éléments de parure et des armatures de flèches.) chronologiquement homogène, comprend six pointes de flèches du type « en sapin » tirées des chailles locales ou régionales, une plus grande foliacée à crans latéraux, malheureusement incomplète (Fig. $n^{\circ} 8$ : Cinq des six pointes de flèches du type « en sapin » et une grande, incomplète, à crans latéraux (longueur conservée : 0,045 m).) et trente-cinq éléments de parure parmi lesquels un lot assez original de huit pendeloques tubulaires obtenues aux dépens de la diaphyse d'os creux (faune) (Fig. $\mathrm{n}^{\circ} 9$ : Éléments de parure en matière dure animale : pendeloques tubulaires en os (longueur de la plus grande : 0,035 m), canines percées, lamelle de défense de sanglier, phalange distale.) . Pour l'industrie lithique, il apparaît que l'armature foliacée à crans latéraux et à retouches bifaciales couvrantes précède souvent la généralisation du type « en sapin ». Ce qui accréditerait l'idée d'une durée de fonctionnement de la tombe plutôt longue. D'utiles rapprochements peuvent être faits avec les sites funéraires environnants tant en grotte [vaste cavité des Baumes Chaudes en aval - fouillée au XIXe s. par le docteur Prunières elle a livré plusieurs centaines d'individus -, sa voisine de Girons et, un peu plus loin près d'Almières, la grotte de la Fromagère - également fouillée par le docteur Prunières (Simanjuntak ; Harry Truman. 1998.) elle abritait un des rares outils connus sur os humain : un poinçon façonné dans l'extrémité distale d'un radius] que sous dolmen (les deux dolmens des Cayroux sur le plateau au nord, de celui de Serres décalé au nord-ouest et les deux de Roudils-Almières au couchant). Le Groupe des Treilles est connu pour sa curieuse pratique de la trépanation. Des témoignages de tels actes d'une audace inouie proviennent des grottes des Baumes Chaudes et de la Fromagère. Ces deux gisements se distinguent également par des blessures par flèches.

7 La base du remplissage de la partie reculée de la cavité (Fig. $n^{\circ} 5$ : Plan de localisation des tessons d'une coupe chasséenne.) a livré une vingtaine de tessons se rattachant, très certainement, à un seul récipient du Néolithique moyen. Il s'agit d'une coupe en calotte 
de sphère de $0,20 \mathrm{~m}$ de diamètre environ. Un sillon interne à profil en $\mathrm{V}$ souligne le bord à lèvre arrondie (Fig. $\mathrm{n}^{\circ} 7$ : Bord de coupe en calotte de sphère à sillon interne.). Alors que les chasséens fréquentaient la grotte des Baumes Chaudes située à $2,5 \mathrm{~km}$ à l'ouest, ils ont poussé une incursion jusqu'ici sans qu'il soit pour le moment possible d'en avancer le but : simple halte? dépôt sépulcral initial?

Enfin, du fond de la cavité (Fig. $n^{\circ} 6$ : Plan de localisation des fragments d'une urne du Bronze final.) provient un panneau, cassé en plusieurs morceaux, de la partie supérieure d'une urne modelée sans soins dont le diamètre de la panse doit se situer aux alentours de $0,42 \mathrm{~m}$. Elle possède un bord droit évasé dont il manque la lèvre. Le raccord col-panse est anguleux. La courbure supérieure de la panse est sommairement agrémentée d'une rangée d'impressions obtenues en appliquant très légèrement sur la paroi fraîche l'extrémité d'une baguette. Cette urne, en particulier par le galbe bien arrondi de la partie haute de la panse, doit se placer au stade terminal du Bronze final ou au tout début de l'âge du Fer. La raison de cette ultime fréquentation protohistorique nous échappe (réserve à provisions (?) dans une fosse creusée dans le niveau sépulcral antérieur) d'autant qu'on ne connait pas pour le moment de site contemporain dans le proche voisinage. Il faut attendre le plein âge du Fer pour voir les caps des environs («Clapas de la Truque » en amont et «Point Sublime » en aval) compléter leur défense naturelle par l'adjonction d'un rempart.

\section{BIBLIOGRAPHIE}

Simanjuntak, Harry Truman. 1998 : Étude de la Collection du Dr Prunières, Contribution à l'étude de la Préhistoire et de la Protohistoire de Lozère et des Grands Causses, Centre de recherche et de documentation préhistorique de Lozère, Banassac-la Canourgue, 528 p.

\section{ANNEXES}


Fig. $\mathrm{n}^{\circ} 1$ : Topographie de la grotte du Valat del Malciapo; plan et coupes. En grisé, la zone sépulcrale.

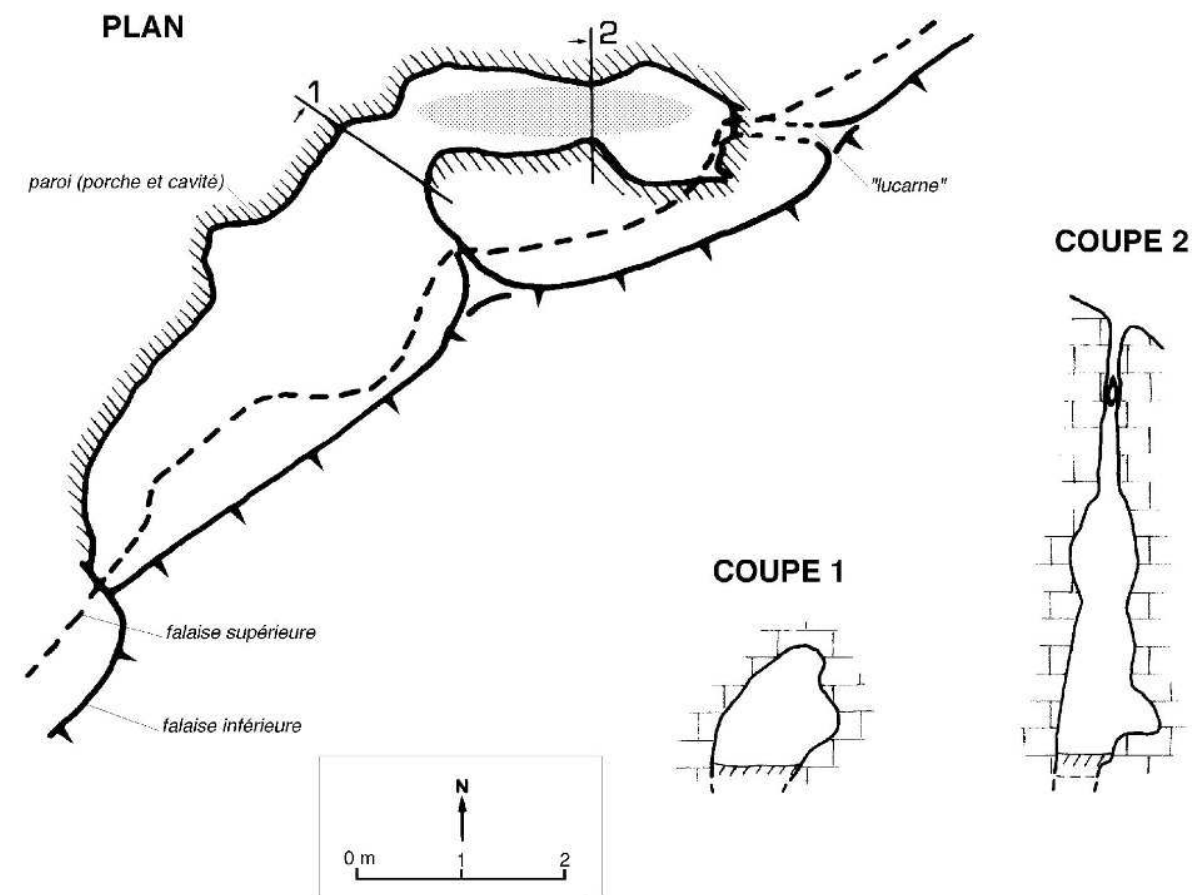

Auteur(s) : Fages, Gilbert. Crédits : ADLFI (2004)

Fig. $n^{\circ} 2$ : Le dépôt sépulcral occupe la partie reculée de la cavité, des vestiges ont migré dans le couloir d'accès en pente vers l'extérieur.

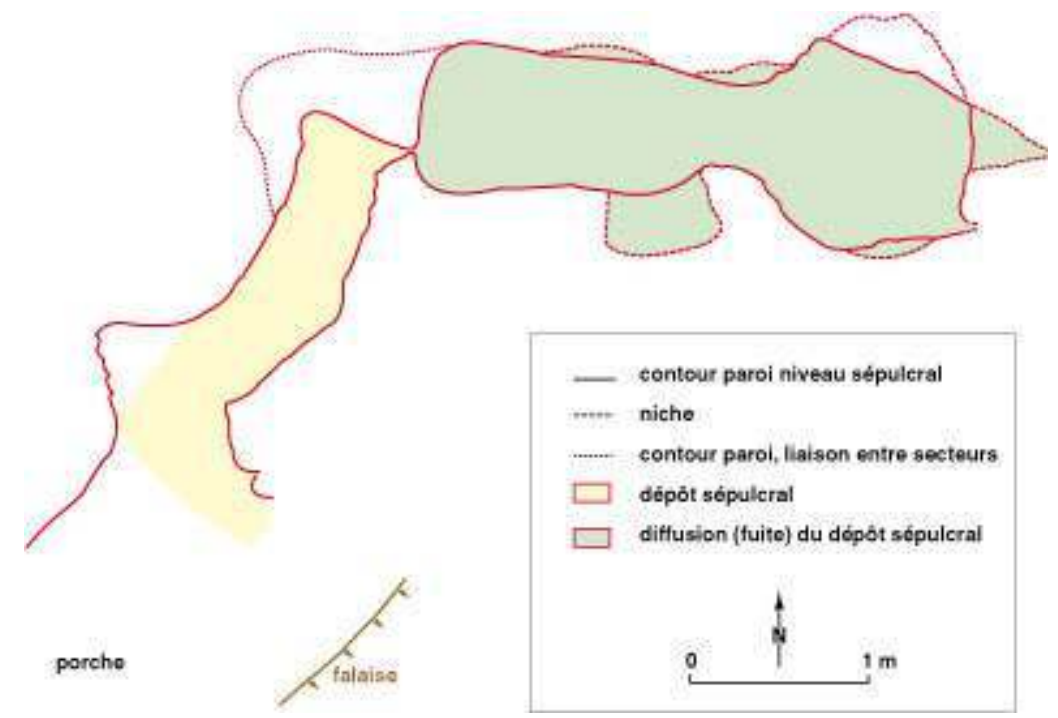

Auteur(s) : Fages, Gilbert. Crédits : ADLFI (2004) 
Fig. $n^{\circ} 3$ : Localisation des mandibules et des principaux fragments crâniens.

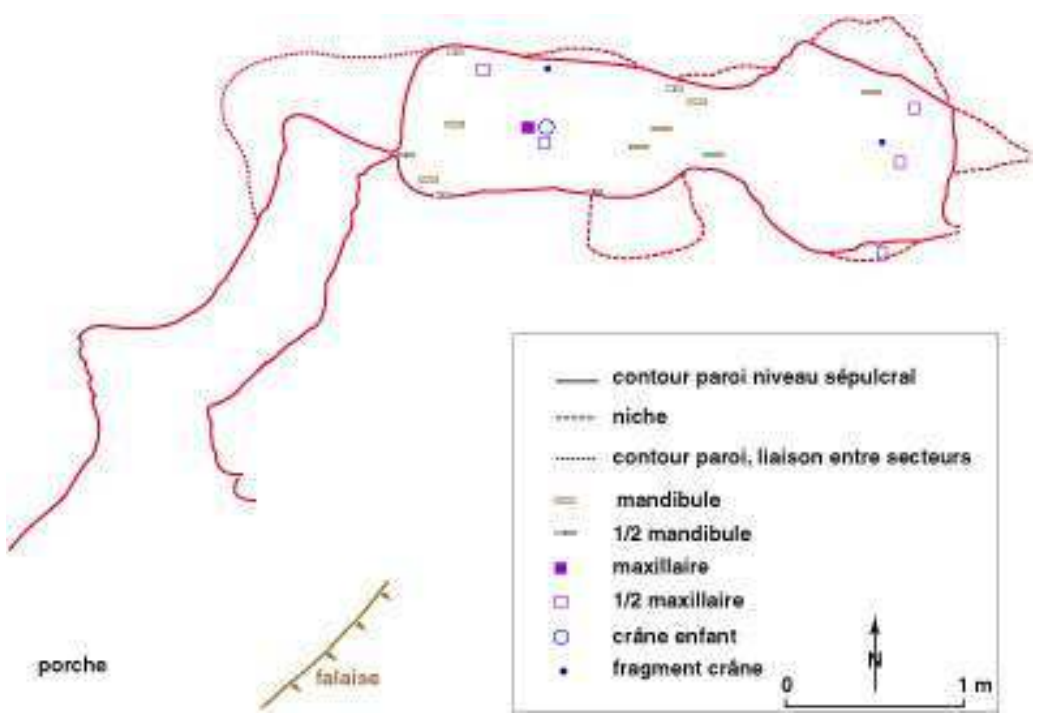

Auteur(s) : Fages, Gilbert. Crédits : ADLFI (2004)

Fig. $n^{\circ} 4$ : Plan de localisation des éléments de parure et des armatures de flèches.

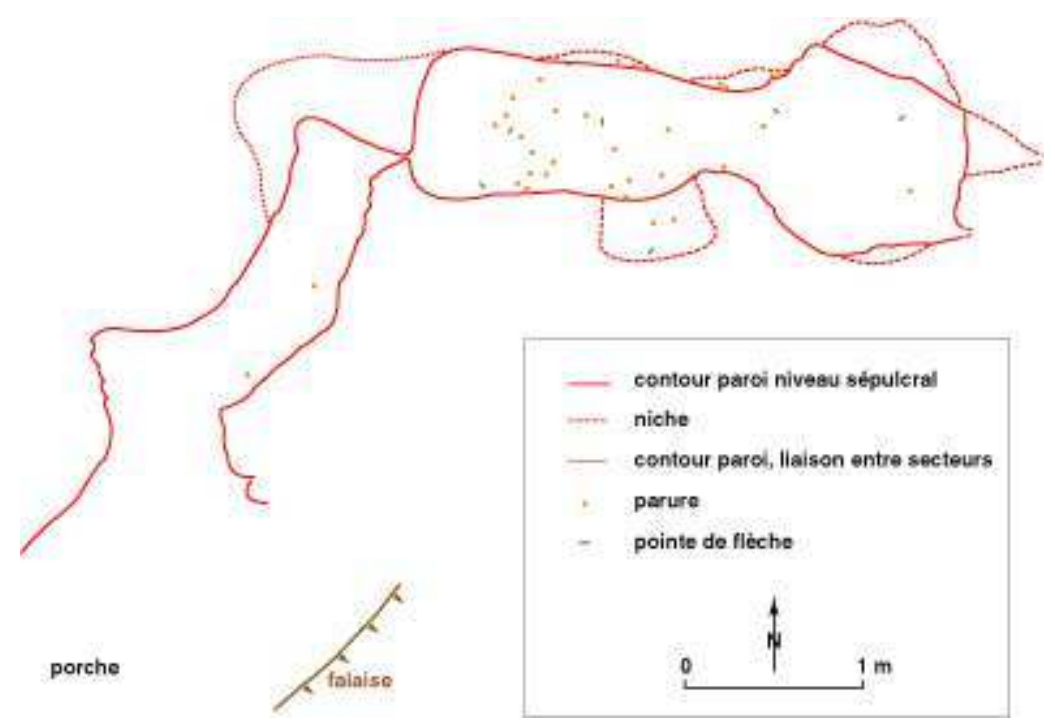

Auteur(s) : Fages, Gilbert. Crédits : ADLFI (2004) 
Fig. $n^{\circ} 5$ : Plan de localisation des tessons d'une coupe chasséenne.

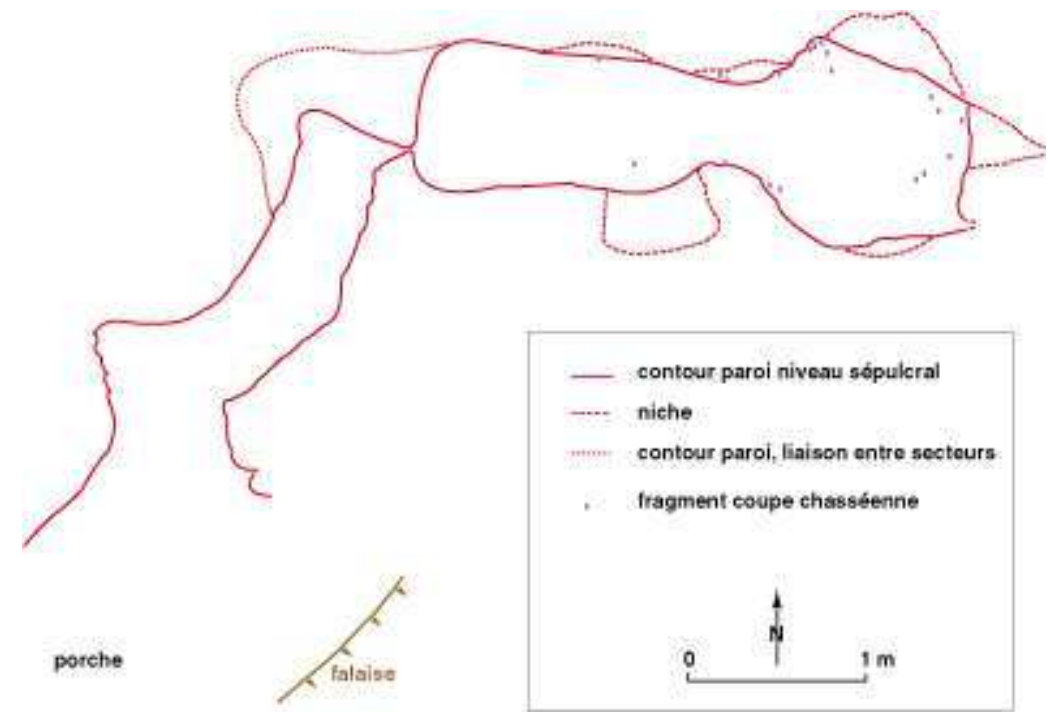

Auteur(s) : Fages, Gilbert. Crédits : ADLFI (2004)

Fig. $n^{\circ} 6$ : Plan de localisation des fragments d'une urne du Bronze final.

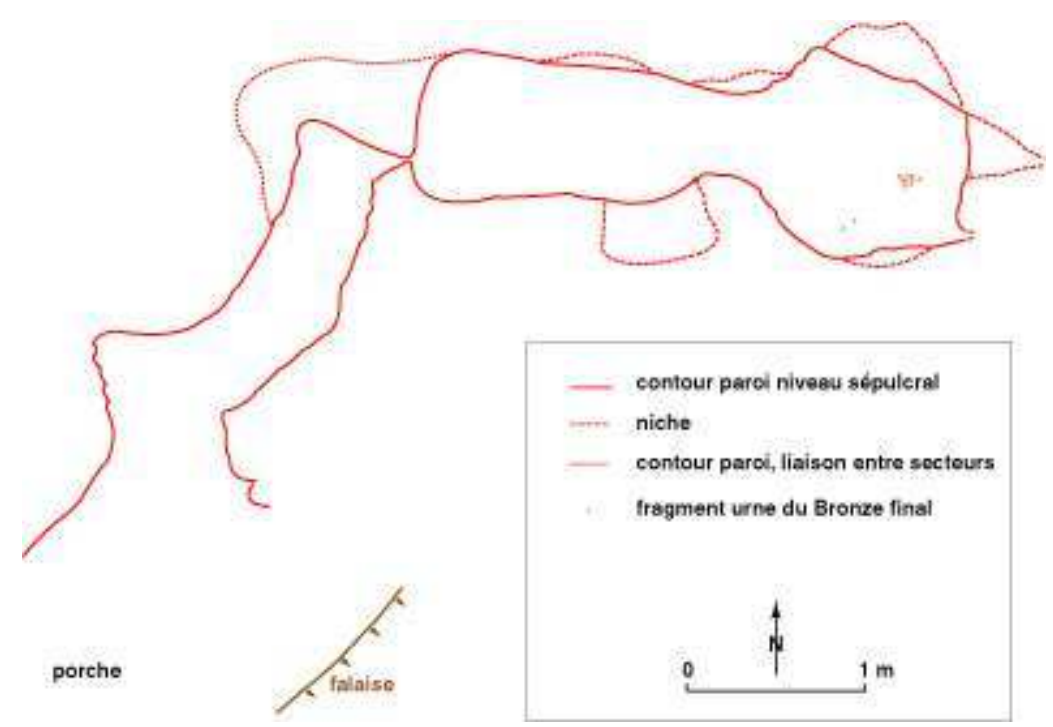

Auteur(s) : Fages, Gilbert. Crédits : ADLFI (2004) 
Fig. $n^{\circ} 7$ : Bord de coupe en calotte de sphère à sillon interne.
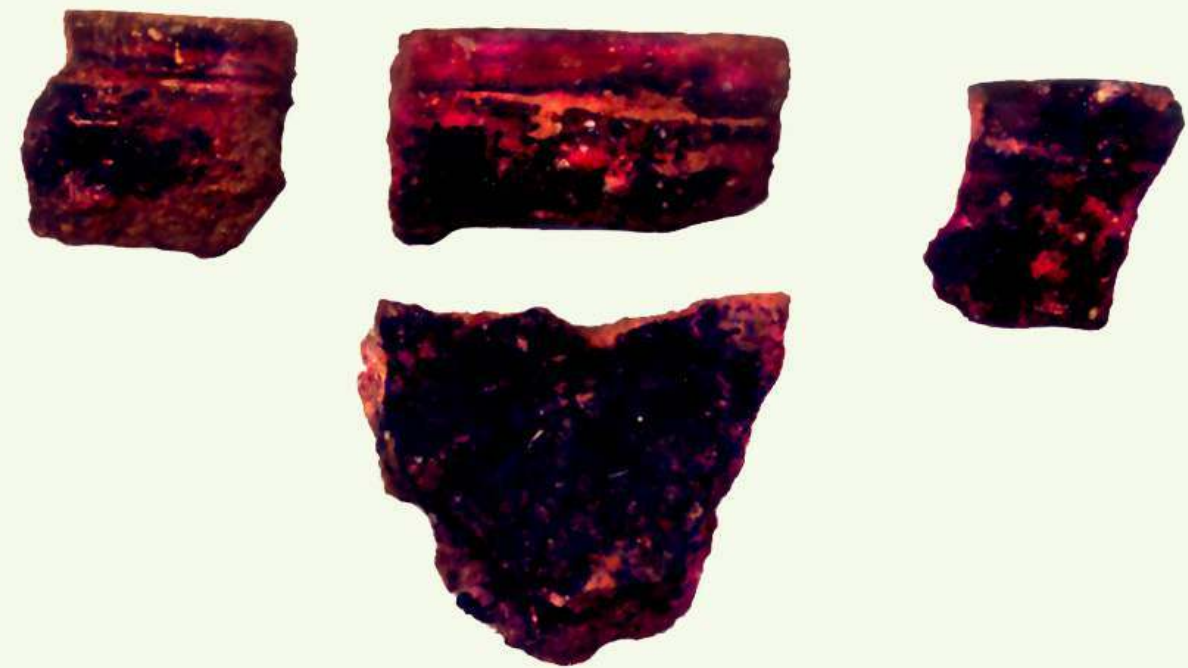

Auteur(s) : Fages, Gilbert. Crédits : ADLFI (2004) 
Fig. $n^{\circ} 8$ : Cinq des six pointes de flèches du type « en sapin » et une grande, incomplète, à crans latéraux (longueur conservée : 0,045 m).

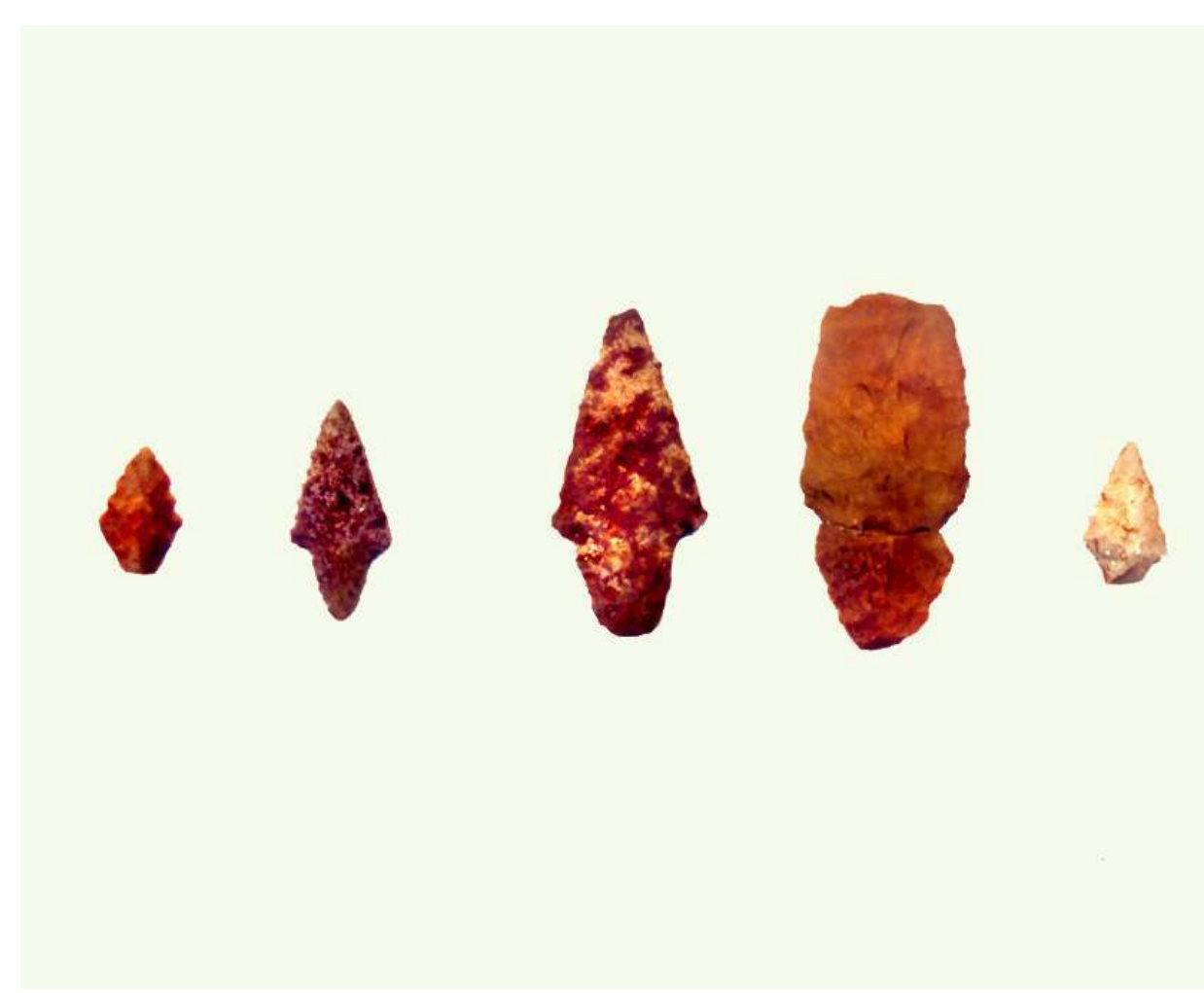

Auteur(s) : Fages, Gilbert. Crédits : ADLFI (2004) 
Fig. $n^{\circ} 9$ : Éléments de parure en matière dure animale : pendeloques tubulaires en os (longueur de la plus grande : 0,035 m), canines percées, lamelle de défense de sanglier, phalange distale.
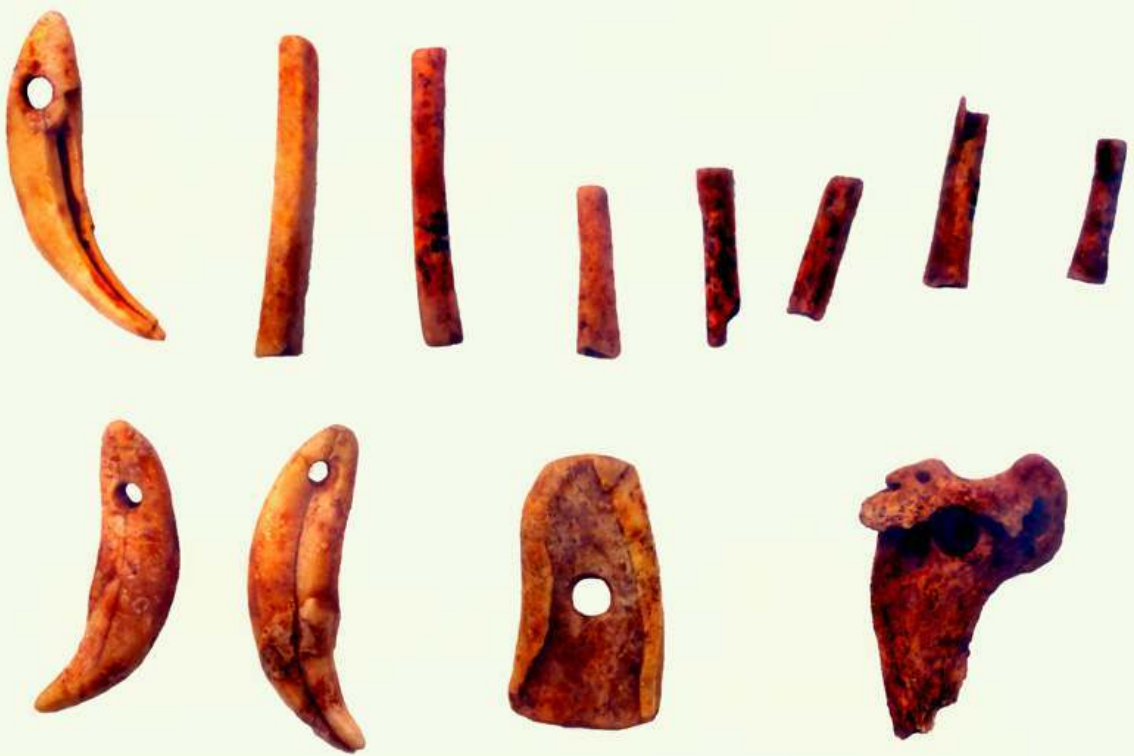

Auteur(s) : Fages, Gilbert. Crédits : ADLFI (2004)

INDEX

Index géographique : Languedoc-Roussillon, Lozère, Saint-Georges-de-Lévéjac operation sauvetage urgent (SU)

\section{AUTEURS}

\section{GILBERT FAGES}

SRA 